\title{
Acquired diaphragmatic hernia in an adult male: A diagnostic challenge
}

\author{
Sachdeva $R,{ }^{1 *}$ Sachdeva $S,{ }^{2}$ Solanki $S^{3}$ \\ ${ }^{1}$ Dept. of TB and Respiratory Medicine, Pt. B.D. Sharma, PGIMS, Rohtak, India. ${ }^{2}$ Dept. of Community Medicine, \\ Pt. B.D. Sharma, PGIMS, Rohtak, India, ${ }^{3}$ ESI, Model Hospital, Ludhiana-141001, India
}

\author{
* Corresponding Author: \\ Dr. Ruchi Sachdeva, DNB, DTCD \\ Dept. of TB and Respiratory Medicine \\ Pt. B.D. Sharma, PGIMS, Rohtak-124001, India \\ Email: drsachdeva@hotmail.com \\ Citation \\ Sachdeva R, Sachdeva S, Solanki S. Acquired \\ diaphragmatic hernia in an adult male: A diagnostic \\ challenge. Nepal Journal of Medical Sciences \\ 2013;2(2):194-6.
}

\begin{abstract}
A rare case of acquired diaphragmatic hernia in a middle aged adult male farmer following occupation related weight lifting is described which was missed at initial presentation. High index of suspicious in conjunct with related investigation can clinch early diagnosis.
\end{abstract}

Keywords: Abdomen; chest; mediastinum; pneumothorax; trauma

\section{Introduction:}

Diaphragm is the most important muscle of respiration that separates thoracic and abdominal cavities. It is composed of peripheral muscular portion which inserts into a central aponeurosis, central tendon. In normal quiet breathing diaphragm is responsible for $75 \%$ of inspiration and inter-costal muscles for rest $25 \%$. Hernias represent a weakness that can evolve into a localized defect, allowing abdominal structures covered with peritoneum to protrude. Diaphragmatic hernias appear secondary to structure or insertion abnormalities of the diaphragm and can be divided in two major categories - congenital and acquired hernias. We describe a rare case of acquired diaphragmatic hernia in a middle aged adult male farmer following occupation related weight lifting.

\section{Case report:}

A 49 year male, average built, chronic smoker, farmer by occupation presented with chief complaint of left sided chest pain and breathlessness since one month. Pain was dull in nature, moderate intensity, non-radiating and aggravated with meal intake. Initially, a diagnosis of left hydropneumothorax was made in the emergency of a private nursing home. An inter-costal chest drain was inserted but subsequently removed as there was no symptomatic relief and referred to us for management. There was no complaint of fever, cough, abdominal pain, vomiting, sweating, palpitation, orthopnea, paroxysmal nocturnal dyspnoea or constipation. However, indigestion was reported. There was no significant past history. His pulse rate was $82 / \mathrm{m}$, blood pressure $120 / 80$, and respiratory rate of $22 / \mathrm{m}$.

Chest examination revealed a mediastinum shift to the right with dull percussion note and a succussion splash on the left side. On auscultation, breath sounds were diminished in intensity on the left upper chest and were found to be absent in the lower zone. Routine blood investigations were unremarkable. Arterial blood gas examination showed mild hypoxemia with partial pressure of arterial oxygen $76 \mathrm{~mm}$ of $\mathrm{Hg}$ on room air. Chest radiograph (Figure 1: PA and left lateral view) showed an air fluid level on left side with 
mediastinal shift to the right.

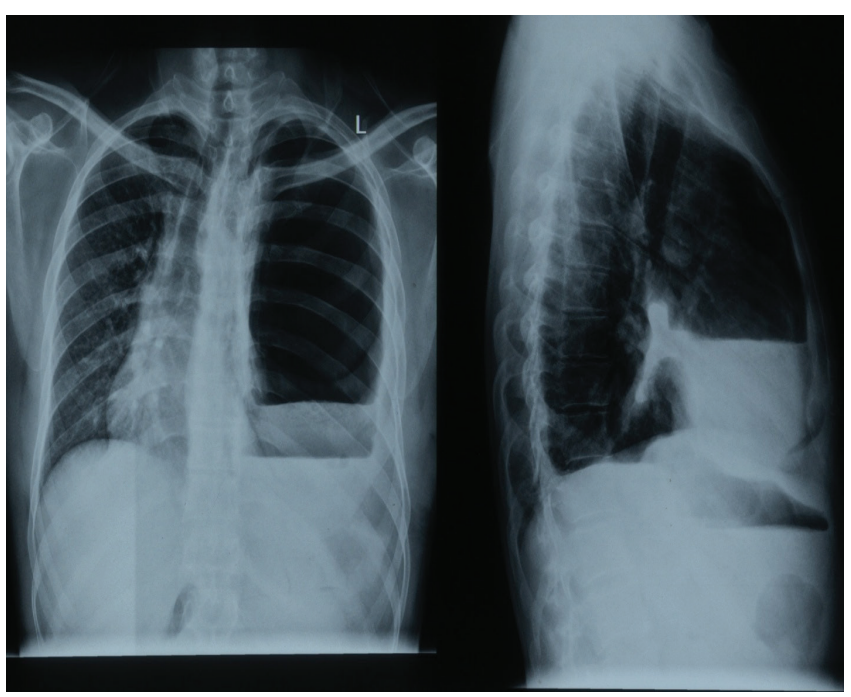

Figure 1: Chest X-ray (PA and left lateral view)

Patient on probing recalled occupation related weight lifting followed by pain in the chest about a month back, subsequent to which all the trail of event precipitated. A provisional diagnosis of spontaneous acquired diaphragmatic hernia was made on chest radiograph after ryle's tube insertion followed by symptomatic and radiological improvement (Figure 2).

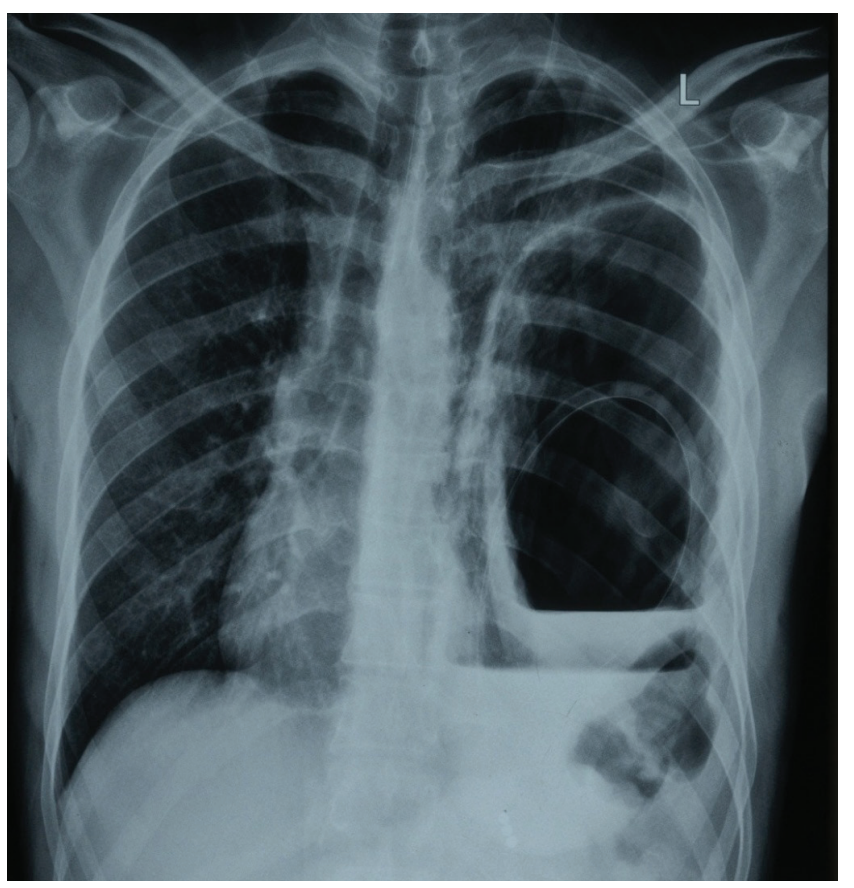

Figure 2: Chest X-ray (PA view following ryles tube insertion)

Computed tomography of chest showed a defect on the left side and herniation of stomach into the thoracic cavity (Figure 3). Patient underwent successful surgical repair with uneventful post-op period and follow up.

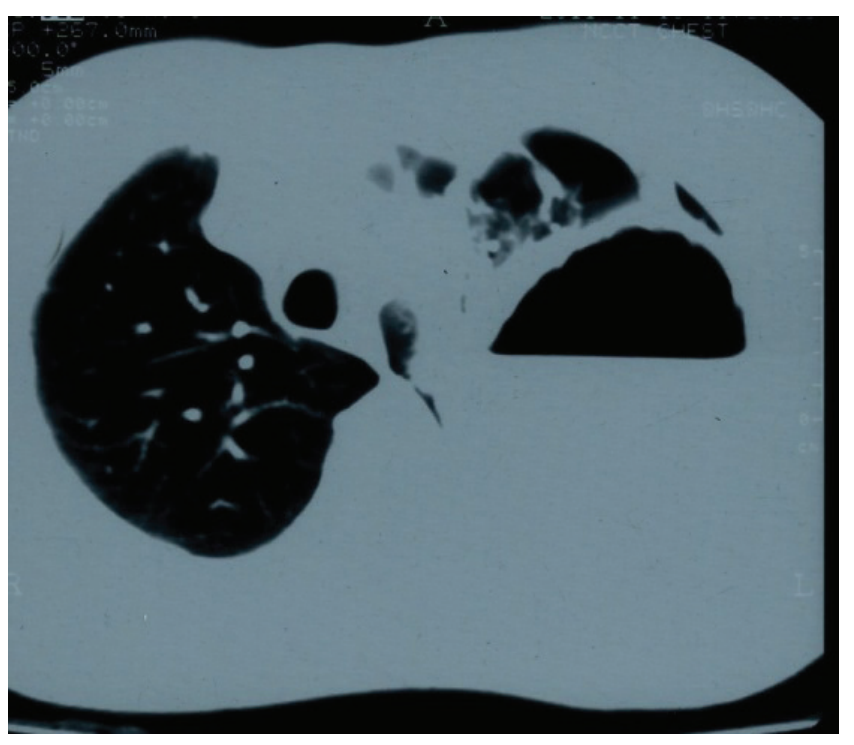

Figure 3: CECT chest showing a defect on the left side of the diaphragm with herniation of stomach into the thoracic cavity

\section{Discussion:}

Acquired diaphragmatic hernias are usually traumatic and may be due to blunt $(0.16 \%-5 \%)$ or penetrating $(12 \%$ $23 \%$ ) thoraco-abdominal trauma with male to female ratio of 4:1 and usually present during third decade of life. ${ }^{1-4}$ However, spontaneous acquired diaphragmatic hernias have also been reported as a rare entity. They are most commonly found at the esophageal hiatus or at the points of failure of embryonic fusion of diaphragm. The latter are usually sub costal (foramen of Morgagni, Larrey's spaces) or posterior (pleuroperitoneal or foramen of Bochdalek) in origin. ${ }^{5} \mathrm{~A}$ 'spontaneous' rupture implies absence of trauma, but there is always a possibility that a diaphragmatic defect arose from some forgotten trauma in the past or structural defect that gave in under strain. ${ }^{6}$ Such occurrence has been reported in athletes, dancer, weightlifter, ${ }^{6}$ during exercise, ${ }^{7}$ eclampsia, labour, ${ }^{8}$ violent emesis, asthma and even pertusis. Spontaneous rupture is rare and may be difficult to diagnose due to absence of typical symptoms. In the present case, a sudden increase in intra abdominal pressure while lifting weight was the likely cause. A remote possibility of a small congenital diaphragmatic hernia may present in adulthood when it remains undetected during childhood.

Various theories put forth for explanation of rupture aretearing of stretched membrane, avulsion of the diaphragm from its point of attachment and sudden force transmission through viscera acting as a viscous fluid. The stomach, colon, liver, spleen and small bowel are most commonly herniated organs. Approximately $69 \%$ of hernias are 
left-sided, 24\% are right-sided, and 15\% are bilateral., ${ }^{9,10}$ Congenital weakness of the diaphragm on the left side and the protective effect of liver on the right side are thought to be the reasons for this phenomenon. ${ }^{11}$

Although examination findings of decreased breath sounds, gurgling sound on auscultation and contra lateral shifting of heart beat can lead to suspicion of diaphragmatic injury, their accuracy in various studies is disappointing. ${ }^{12}$ Findings strongly suggestive of diaphragmatic rupture in the chest radiograph include: (i) an abnormal, usually high diaphragmatic contour with an arch like shadow that simulates the diaphragm; (ii) gas bubbles or air-fluid levels in the chest above the expected level of the diaphragm; (iii) intra-thoracic abdominal viscera, with or without a site of focal constriction (collar sign); and (iv) clear demonstration of the nasogastric tube tip above the left hemi diaphragm. ${ }^{13}$ Ultrasonography can assess the extent of herniated organs into the thoracic cavity with positive predictive value of $88 \%$. Computed tomography with a sensitivity of $14 \%$ to $82 \%$ and specificity of $87 \%$ is also helpful for detecting the defect. ${ }^{1,14}$ Differential diagnosis includes, giant hiatal hernias, congenital diaphragmatic hernias, pulmonary sequestration, neoplasias, phrenic nerve palsy, atelectasis, sub-pulmonic effusion, sub-pulmonic abscess and eventration of the diaphragm. ${ }^{15}$ Diverse presentations (emergency to asymptomatic) of diaphragmatic hernias have been noticed but the condition is frequently overlooked inspite of widespread awareness.

Conclusion: In conclusion, high index of suspicious in conjunct with appropriate investigation can clinch early diagnosis and help in management of this rare condition.

Acknowledgement: Dept. of TB, Respiratory Medicine and Radio-diagnosis, Pt. B.D. Sharma, PGIMS,

Rohtak-124001, India.

\section{Conflict of interest: none}

funding: nil

\section{References:}

1. Sliker CW. Imaging of diaphragm injuries. Radiol Clin North Am 2006;44:199-211.

2. Schumpelick V, Steinau G, Schluper I, et al. Surgical embryology and anatomy of the diaphragm with surgical applications. Surg Clin North Am 2000;80:213-39.
3. Rashid F, Chakrabarty MM, Singh R, et al. A review on delayed presentation of diaphragmatic rupture. World J Emerg Surg 2009;4:32.

4. Chughtai T, Ali S, Sharkey P, et al. Update on managing diaphragmatic rupture in blunt trauma: a review of 208 consecutive cases. Candian J Surg 2009;52:177-81.

5. Poe RH, Schowengerdt CG. Two cases of a traumatic herniation of the liver. Am Rev Respir Dis 1972;105:959-63.

6. Jha P, Hutchinson S, Spychal B, et al. Respiratory distress after heavy lifting. J R Soc Med 2004;97:290-1.

7. Bisgaard C, Rodenberg JC, Lundgaard J. Spontaneous rupture of the diaphragm. Scand J Thorac Cardiovasc Surg 1985;19:177-80.

8. Dave KS, Bekassy SM, Wooler GH, et al. Spontneous rupture of the diaphragm during delivery. Br J Surg 2005;60:666-8.

9. Kuhlman JE, Pozniak MA, Collins J, et al. Radiographic and CT findings of blunt chest trauma: aortic injuries and looking beyond them. Radiographics 1998;18:1085-6.

10. Feliciano DV, Cruse PA, Mattox KL, et al. Delayed diagnosis of injuries to the diaphragm after penetrating wounds. J Trauma 1988;28:1135-44.

11. Lucido JL, Wall CA. Rupture of the diaphragm due to blunt trauma. Arch Surg 1963;86:989-99.

12. Moore JB, Moore EE, Thompson JS. Abdominal injuries associated with penetrating trauma in the lower chest. Am J Surg 1980;140:724-30.

13. Dhruva C, Kadian YS, Fotedar S, et al. Ttraumatic rupture of diaphragm with flail chest mimicking tension hydropneumothorax. Ind J Chest Dis Allied Sci 2009;51:173-5.

14. Patselas TN, Gallagher EG. The diagnostic dilemma of diaphragm injury. Am Surg 2002;68:633-9.

15. Oh KS, Newman B, Bender TM, et al. Radiologic evaluation of the diaphragm. Radiol Clin North Am 1988;26:355-64. 